University of Nebraska - Lincoln

DigitalCommons@University of Nebraska - Lincoln

Faculty Papers and Publications in Animal

Science

Animal Science Department

June 2005

\title{
Epigenetic Transgenerational Actions of Endocrine Disruptors and Male Fertility
}

\author{
Matthew D. Anway \\ Washington State University, Pullman \\ Andrea S. Cupp \\ University of Nebraska-Lincoln, acupp2@unl.edu \\ Mehmet Uzumcu \\ Rutgers University \\ Michael K. Skinner \\ Washington State University, Pullman
}

Follow this and additional works at: https://digitalcommons.unl.edu/animalscifacpub

Part of the Animal Sciences Commons

Anway, Matthew D.; Cupp, Andrea S.; Uzumcu, Mehmet; and Skinner, Michael K., "Epigenetic

Transgenerational Actions of Endocrine Disruptors and Male Fertility" (2005). Faculty Papers and Publications in Animal Science. 169.

https://digitalcommons.unl.edu/animalscifacpub/169

This Article is brought to you for free and open access by the Animal Science Department at DigitalCommons@University of Nebraska - Lincoln. It has been accepted for inclusion in Faculty Papers and Publications in Animal Science by an authorized administrator of DigitalCommons@University of Nebraska - Lincoln. 


\section{Epigenetic Transgenerational Actions of Endocrine Disruptors and Male Fertility}

\author{
Matthew D. Anway, Andrea S. Cupp, ${ }^{*}$ Mehmet Uzumcu, ${ }^{\dagger}$ \\ and Michael K. Skinner $¥$
}

\author{
Center for Reproductive Biology, School of Molecular Biosciences, \\ Washington State University, Pullman, WA 99164-4231 \\ *Present address: Department of Animal Science, University of Nebraska-Lincoln, \\ Lincoln, NE 68583-0908; † Present address: Department of Animal Science, \\ Rutgers University, 84 Lipman Drive, New Brunswick, NJ 08901-8525 \\ ‡ Corresponding author — email skinner@mail.wsu.edu \\ Submitted December 2, 2004; accepted March 22, 2005
}

\begin{abstract}
Transgenerational effects of environmental toxins require either a chromosomal or epigenetic alteration in the germ line. Transient exposure of a gestating female rat during the period of gonadal sex determination to the endocrine disruptors vinclozolin (an antiandrogenic compound) or methoxychlor (an estrogenic compound) induced an adult phenotype in the $F_{1}$ generation of decreased spermatogenic capacity (cell number and viability) and increased incidence of male infertility. These effects were transferred through the male germ line to nearly all males of all subsequent generations examined (that is, $F_{1}$ to $F_{4}$ ). The effects on reproduction correlate with altered DNA methylation patterns in the germ line. The ability of an environmental factor (for example, endocrine disruptor) to reprogram the germ line and to promote a transgenerational disease state has significant implications for evolutionary biology and disease etiology.
\end{abstract}

Treatments, such as irradiation and chemotherapy, and compounds, such as environmental toxins, pose a threat to the integrity of the genome. Studies have shown that these agents can result in genetic or developmental defects in the offspring or $\mathrm{F}_{1}$ generation from an exposed gestating mother. The ability of an external agent to induce a transgenerational effect requires stable chromosomal alterations or an epigenetic phenomenon such as DNA methylation (1). In the present study, transgenerational refers to a germline transmission to multiple generations, minimally to the $F_{2}$ generation. Transgenerational effects of irradiation were the first to be identified through transmission of DNA mutations in the germ line to multiple generations (2), often associated with tumor formation. Chemotherapeutic treatments (3) and environmental toxins such as endocrine disruptors (4) can cause effects in the $F_{1}$ generation, but they have not been shown to affect the $\mathrm{F}_{2}$ generation. Although no effects have been shown to be transgenerational, the potential impact of such transgenerational effects of endocrine disruptors has been discussed (5).

Epigenetic alterations that could lead to transgenerational transmission of specific genetic traits have recently been identified ( 1 , 6). A transgenerational phenotype or genetic trait requires a permanent reprogramming of the germ line. During mammalian germ cell development the methylation state of the genome is reprogrammed. As primordial germ cells (PGCs) migrate down the genital ridge, a demethylation starts and is complete on colonization in the early gonad ( 7 , 8 ). Germ cells in the gonad then undergo remethylation in a sex-specific manner during gonadal sex determination (9). Although demethylation may not require the gonadal somatic cells, remethylation of the germ line appears to be dependent on association with the somatic cells in the gonads (7). Gonadal sex determination and testis development occur between embryonic days 12 and 15 (E12 to E15) in the rat (after midgestation in the human) and are initiated by the differentiation of precursor Sertoli cells in response to the testis-determining factor Sry. Aggregation of the precursor Sertoli cells, PGCs, and migrating mesonephros cells (precursor peritubular myoid cells) promotes testis morphogenesis and cord formation (10, 11). During the period of gonadal sex determination, the fetal testis contains steroid receptors and is a target for endocrine agents. The androgen receptor (AR) and estrogen receptor $-\beta(E R \beta)$ are present in Sertoli cells, precursor peritubular myoid cells, and germ cells at the time of cord formation (E14) $(12,13)$. Although steroids are not produced by the testis at this stage of development, estrogenic and androgenic substances have the ability to influence early testis cellular functions. Therefore, steroidal factors acting inappropriately at the time of gonadal sex determination potentially could reprogram the germ line through an epigenetic mechanism (altered DNA methylation) to cause the transgenerational transmission of an altered phenotype or genetic trait.

The estrogenic and antiandrogenic endocrine disruptors used in the current study are methoxychlor and vinclozolin, respectively. Vinclozolin is a commonly used fungicide in the wine industry that is metabolized into more active (i.e., higher affinity binding to androgen receptor) compounds (14). Methoxychlor is used as a pesticide to replace DDT and is metabolized into active compounds with the ER $\alpha$ agonist, the ER $\beta$ antagonist, and anti-androgenic activity (15-17). Vinclozolin or methoxychlor exposure in the late embryonic or early postnatal period influences sexual differentiation, gonad formation, and reproductive functions in the $\mathrm{F}_{1}$ generation (14, 18, 19). Transient exposure (daily intraperitoneal injection of 100 or $200 \mathrm{mg} / \mathrm{kg}$ dose) of a gestating female rat to methoxychlor or vinclozolin between E8 and E15 promotes reduced spermatogenic capacity associated with increased spermatogenic cell apoptosis and decreased sperm number and motility in the adult $\mathrm{F}_{1}$ generation $(20,21)$. A similar exposure between E15 and E20 had no effect on the $\mathrm{F}_{1}$ generation testis $(20,21)$. These observations were extended in the present study by treating the gestating mother with vinclozolin. $F_{1}$ generation male rats were mated with $\mathrm{F}_{1}$ generation females from different litters. Subsequent breeding continued for four generations with sufficient numbers of animals to avoid sibling inbreeding. Adult males from $\mathrm{F}_{1}, \mathrm{~F}_{2}, \mathrm{~F}_{3}$, and $\mathrm{F}_{4}$ generations between postnatal days PND60 and PND180 were killed. Testes were isolated for histological examination, and caudal epididymal sperm were collected for sperm counts and motility measurements. Only the original gestating mother $\left(\mathrm{F}_{0}\right)$ of the $\mathrm{F}_{1}$ generation received a transient endocrine disruptor treatment. Control groups of animals were bred in a similar manner after vehicle treatment (dimethylsulfoxide buffer alone injected) of the $\mathrm{F}_{0}$ gestating mother. Analysis of cellular apoptosis demonstrated a greater than two-fold increase in spermatogenic cell apoptosis in the vinclozolin treatment animals for the $F_{1}$ to $F_{4}$ generations (Figure 1A). Sperm numbers were reduced minimally, $20 \%$, and sperm forward motility was reduced about 25 to $35 \%$ for vinclozolin generation animals (Figure 1, B and C). More than $90 \%$ of all males analyzed from all generations had the germ cell defect of increased spermatogenic cell apoptosis. Therefore, the frequency of the phenotype was $>90 \%$ and did not decline between the $\mathrm{F}_{1}$ and $\mathrm{F}_{4}$ generations. A similar experiment was performed with methoxychlor. After transient embryonic methoxychlor exposure (E8 to E15), a similar phenotype was observed in both the $\mathrm{F}_{1}$ and $\mathrm{F}_{2}$ animals (fig. S1). Therefore, both vinclozolin and methoxychlor induced transgenerational defects in spermatogenic capacity and sperm viability. 
An outcross experiment was performed to determine whether the transgenerational phenotype was transmitted through the male germ line. Vinclozolin $F_{2}$ generation males (i.e., male progeny from an $\mathrm{F}_{0}$ treated mother) were crossed with wild-type untreated control females, and the offspring were analyzed. The vinclozolin outcross (VOC) male progeny also had an increase in spermatogenic cell apoptosis and a decrease in sperm number and motility (Figure 1). The reverse vinclozolin outcross (RVOC) with vinclozolin $\mathrm{F}_{2}$ generation females and wild-type control males demonstrated no effect on the spermatogenic cells (Figure 1). Therefore, the endocrine disruptorinduced transgenerational phenotype appears to be transmitted through the male germ line.

The morphology of the testes from control and treated rats was similar for all animals examined on PND60 in all $F_{1}$ to $F_{4}$ vinclozolin generations. Periodically, male rats older than 90 days of age developed complete infertility associated with small testis and severely reduced spermatogenesis, which was not seen in the PND60 animals. This occurred in 4 out of a total of $50 \mathrm{~F}_{1}, \mathrm{~F}_{2}, \mathrm{~F}_{3}$, and $\mathrm{F}_{4}$ generation animals. Therefore, $8 \%$ of the vinclozolin transgenerational males developed complete infertility. None of the 42 control $F_{1}$ to $F_{4}$ generation animals were infertile. The testis histology for a representative infertile vinclozolin $\mathrm{F}_{3}$ generation animal is shown in Figure 2 and demonstrates a loss of normal spermatogenesis (no germ cells present) and abnormal seminiferous tubule morphology. The control $\mathrm{F}_{3}$ male showed normal morphology with normal spermatogenesis. Although most of the animals older than 90 days of age were fertile, $\sim 20 \%$ developed a dramatic decrease in spermatogenic capacity $(50 \%$ of the tubules having impaired germ cell development) in all the generations examined. The VOC males also had increased infertility in $\sim 20 \%$ of the animals over 90 days of age. The treated males that were fertile showed no change in litter size, newborn pup weights, or testis weight per body weight when compared with the control animals of any of the $F_{1}$ to $F_{4}$ generations examined. Nearly all the treated male progeny had the minimal phenotype of twofold increase in spermatogenic cell apoptosis, and the majority had a decrease in epididymal sperm number (Figure 1, A and B).

The transmission of this endocrine disruptor-induced testis phenotype in a transgenerational manner suggests an epigenetic alteration of the male germ line. The frequency observed for the phenotype ( $>90 \%$ of all males in all generations) cannot be explained through genetic DNA sequence mutational mechanisms. A high-frequency genetic (DNA sequence) mutation hotspot event would be an order of magnitude less than that observed $(2,22,23)$. Subsequent segregation of this mutation would likely result in a reduction of the phenotype frequency with each generation. In addition, the random nature of genetic (DNA sequence) mutations is generally more variable in the phenotype observed $(2,22,23)$. In contrast, an epigenetic mechanism involving reprogramming of the germ line could result in the high frequency observed. In addition, the developmental period

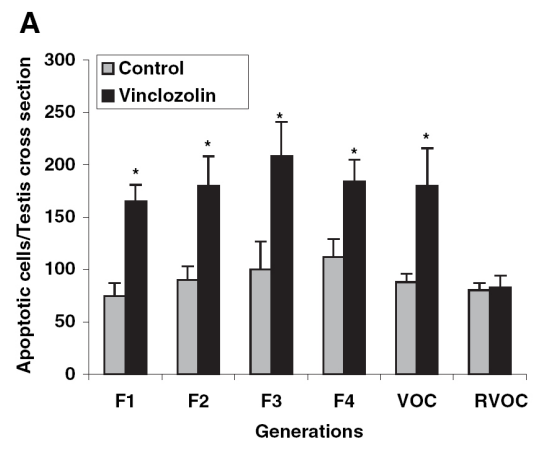

Figure 1. Transgenerational phenotype after vinclozolin treatment of $F_{0}$ gestating mothers. (A) Spermatogenic cell apoptosis, (B) epididymal sperm counts, and (C) epididymal sperm motility in PND60 to 180 control and vinclozolin off-spring Sprague-Dawley rats in the $F_{1}$, $F_{2}, F_{3}$, and $F_{4}$ generations, and vinclozolin $F_{2}$ generation male outcross (VOC) to wild-type control females, and vinclozolin $\mathrm{F}_{2}$ generation female reverse outcross (RVOC) to wild-type control males. Statistically significant differences between control and vinclozolin treatment generations are indicated by $\left(^{*}\right)$ for $P<$ 0.001 with a two-way analysis of variance test. The $n$ value for each bar ranged between 10 and 30 animals. Detailed methods are provided in Supporting Materials (below).
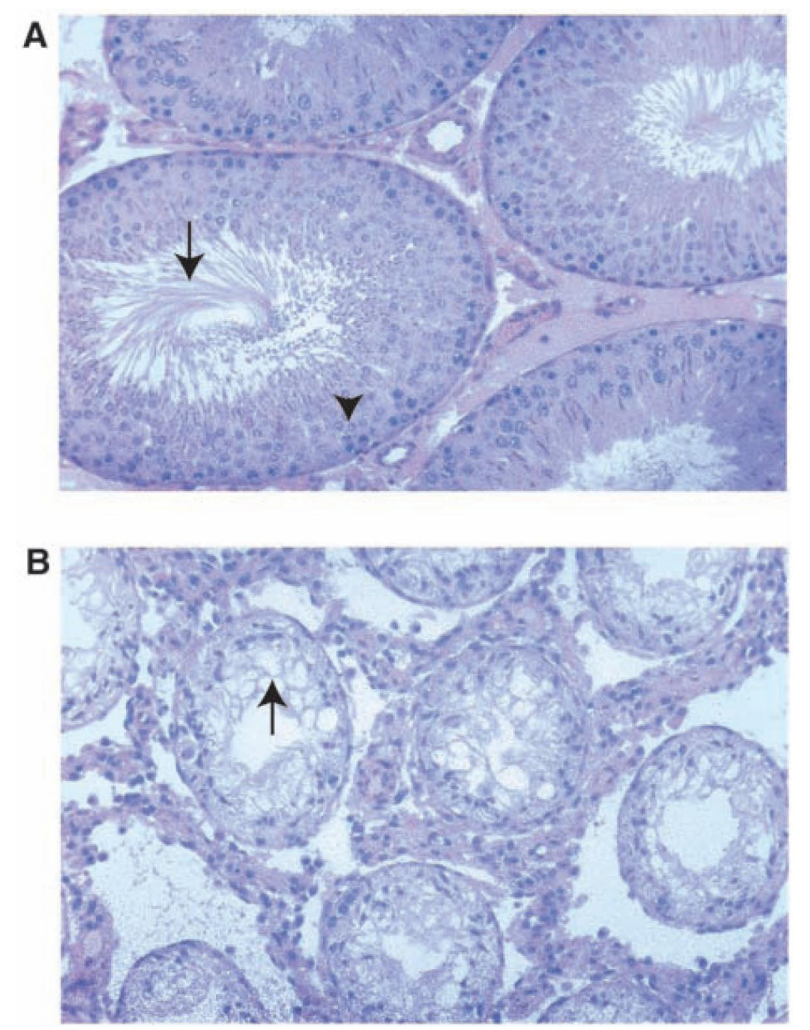

used for the endocrine disruptor exposure was during the remethylation programming of the germ line. Although we cannot exclude the possibility of a novel genetic (DNA sequence) mutational event, available information suggests an epigenetic mechanism is involved.
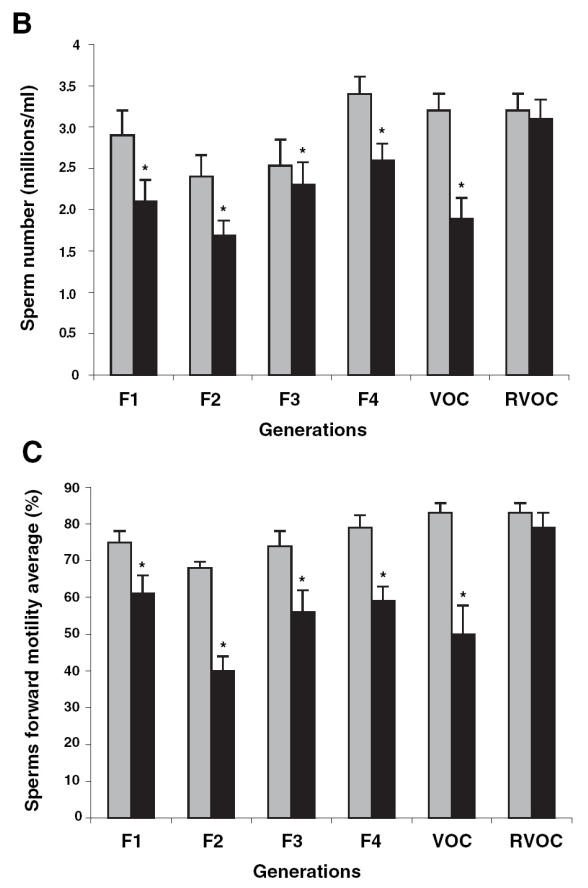

Figure 2. Testis histology from control (A) and vinclozolin treatment (B) 100-dayold $\mathrm{F}_{3}$ generation animals, $x$ 200 magnification. The vinclozolin $\mathrm{F}_{3}$ generation male is a representative infertile male. Arrow in $(A)$ identifies the tails of elongate spermatozoa in the seminiferous tubule lumen; arrowhead labels spermatocytes in the tubule epithelial layer. Arrow in (B) identifies the lack of germ cells in the seminiferous tubule. Methods are provided in Supporting Materials (below). 
A

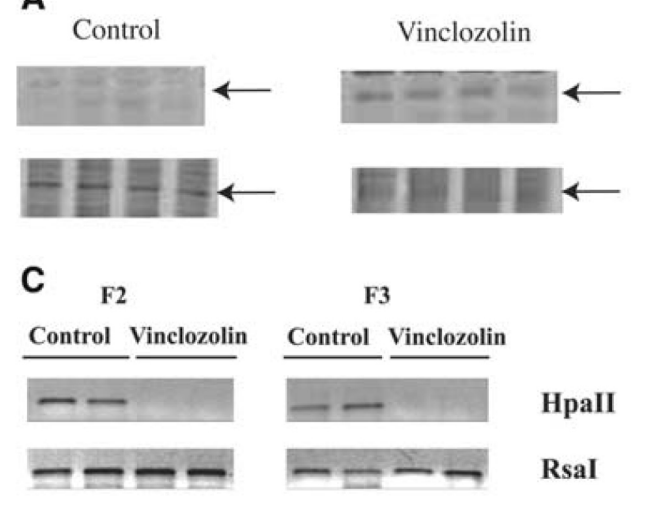

B

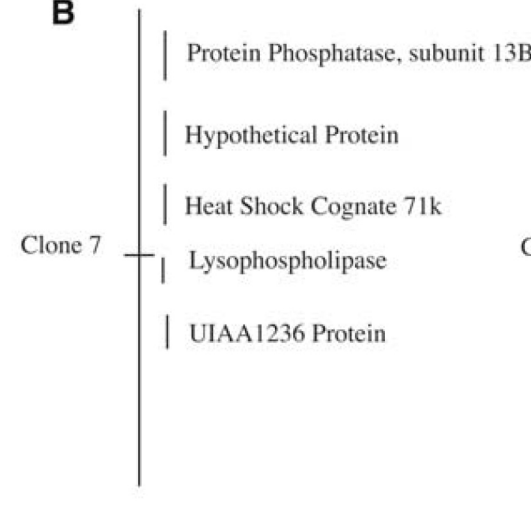

$6 \mathrm{q} 32$

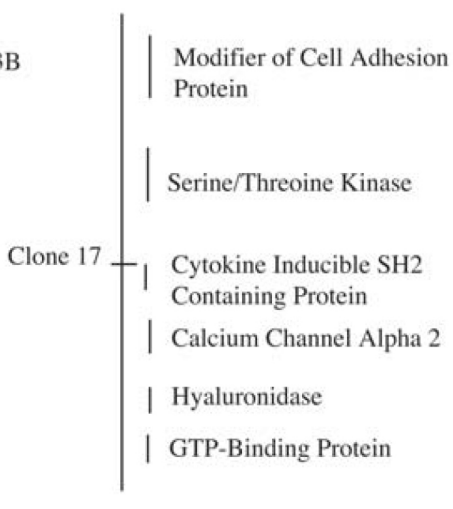

$8 \mathrm{q} 32$

Figure 3. DNA methylation analysis from control and vinclozolin offspring testis. (A) Representative gel images of the PCR-based methylation-sensitive Hpa II restriction enzyme digest analysis with representative band (arrow) affected in PND6 testis from control and vinclozolin treatment animals. Each lane represents a different individual animal $(n=4)$. (B) Location of selected sequences on specific chromosomes for two representative DNA sequences with altered DNA methylation patterns termed clone 7 and 17. (C) Methylation-sensitive restriction enzyme PCR analysis of the methylation state of clone 17 (i.e., cytokine-inducible $\mathrm{SH} 2$ protein) gene in epididymal sperm from $\mathrm{F}_{2}$ and $\mathrm{F}_{3}$ generations from control and vinclozolin-treated animals. The bands presented are representative of sperm DNA collected from different animals from different litters and are consistent in four out of eight $F_{2}$ animals and two out of five $F_{3}$ animals analyzed. Methods are provided in Supporting Materials (below).

The only epigenetic mechanism currently known to influence germline transmission involves the methylation pattern of imprinted genes. This study does not investigate specific known imprinted genes, but focuses on the effects of the endocrine disruptor on the total genome. Fisher rats were used because of reduced polymorphisms, which made methylation studies more consistent and reproducible. PND6 testes were collected from male $F_{1}$ progeny of vinclozolin-treated and control rats. A methylation-sensitive restriction enzyme digestion analysis involving a polymerase chain reaction (PCR)-based procedure was used to assess changes in DNA methylation patterns (Methods in SOM). Animals $(n=4)$ from different litters of control and treated animals were analyzed. The vinclozolin-induced methylation patterns were similar in the replicate animals. About 25 different PCR products were identified that had altered DNA methylation patterns after the endocrine disruptor treatment. A representative change in methylation pattern is shown in Figure 3A and fig. S2. These methylation experiments were extended by isolating and cloning several of the DNA fragments with apparent altered methylation. Two of the DNA fragments were sequenced and mapped to $\mathrm{CpG}$-rich regions on chromosomes $6 \mathrm{q} 32$ and $8 \mathrm{q} 32$ (Figure 3B). Clone 7 mapped to $6 \mathrm{q} 32$ and was within the lysophospholipase (LPLase) gene (accession NM144750). LPLase is a critical enzyme in the synthesis of important bioactive lipids and associated signaling (24). Clone 17 mapped to $8 \mathrm{q} 32$ and is within $1 \mathrm{~kb}$ of the start site of an uncharacterized protein termed cytokine-inducible SH2 protein (accession AJ243907 [GenBank]). Whether the genes identified are causal factors or simply markers (i.e., downstream) of the transgenerational epigenetic pheno-type remains to be determined.

The epigenetic transgenerational transmission of the altered methylation pattern through the male germ line was investigated. PCR primers were designed for the flanking regions of the clone 17 gene, cytokine-inducible SH2 protein and were used to investigate altered methylation with the methylation-sensitive restriction enzyme digest procedure. Epididymal sperm were isolated from vinclozolin treatment $\mathrm{F}_{2}$ and $\mathrm{F}_{3}$ generation animals. As shown in Figure 3C, the control animals had the PCR product, whereas the vinclozolin treatment $\mathrm{F}_{2}$ and $\mathrm{F}_{3}$ animals did not. The control Rsa I digest had a PCR product in all samples. Therefore, the vinclozolin treatment $\mathrm{F}_{2}$ and $\mathrm{F}_{3}$ generation sperm samples appeared to have altered DNA methylation in this clone 17 gene compared with control animal sperm. An alternate bisulfite DNA sequence analysis was used to confirm the methylation changes. Bisulfite analysis confirmed the altered methylations of the LPLase gene within the $\mathrm{CpG}$ island identified. This bisulfite altered sequence (fig. S3) was observed in $\sim 25 \%$ of the vinclozolin treatment sperm DNA samples analyzed. A single-gene methylation event alone is not likely sufficient to promote the phenotype, but could cause alterations in a subset of genes. For example, 25 DNA sequences with altered methylation were identified, and if a random subset of genes with altered methylation promotes the phenotype, a $25 \%$ frequency for altered methylation of a single gene would be significant. Observations indicate that the endocrine disruptors can induce an epigenetic transgenerational change in the DNA methylation pattern of the male germ line. The epigenetic alterations observed involve both hypermethylation and hypomethylation events.

Two different endocrine disruptors, vinclozolin and methoxychlor, after a transient embryonic exposure at a critical time during gonadal sex determination (E8 to E15 in the rat), promoted an adult testis phenotype of decreased spermatogenic capacity and male infertility. No gross abnormality was observed in any other tissues examined, and serum testosterone levels were found to be normal in all the animals examined. This phenotype was found to be transgenerational and appears associated with altered DNA methylation of the male germ line. The phenotype was observed in nearly all males from all vinclozolin generations, such that a genetic mutation event (e.g., alteration in DNA sequence) is not likely to be a major factor. The frequency of a genetic mutation would be orders of magnitude less than the transmission frequency observed in the current study $(2,22,23)$. Preliminary data demonstrate no major effect on the female, but a number of abnormal pregnancy outcomes were observed in pregnant females from offspring of vinclozolin-treated animals, but not controls. The abnormal phenotype has similarities to preeclampsia that include death, severe anemia, and blood cell defects. This study shows that environmental factors can induce an epigenetic transgenerational phenotype through an apparent reprogramming of the male germ line. It should be noted that the exposure levels used in these studies are higher than anticipated for environmental exposure; hence, future toxicology studies would be needed to ascertain the possible impact on animal populations. 


\section{References}

1. V. Rakyan, E. Whitelaw, Curr. Biol. 13, R6 (2003).

2. R. Barber, M. A. Plumb, E. Boulton, I. Roux, Y. E. Dubrova, Proc. Natl. Acad. Sci. U.S.A. 99, 6877 (2002).

3. I. D. Morris, Int. J. Androl. 25, 255 (2002).

4. C. M. Foran, B. N. Peterson, W. H. Benson, Toxicol. Sci. 68, 389 (2002).

5. C. DeRosa, P. Richter, H. Pohl, D. E. Jones, J. Toxicol. Environ. Health $B$ Crit. Rev. 1, 3 (1998).

6. V. K. Rakyan et al., Proc. Natl. Acad. Sci. U.S.A. 100, 2538 (2003).

7. P. Hajkova et al., Mech. Dev. 117, 15 (2002).

8. G. Durcova-Hills, J. Ainscough, A. McLaren, Differentiation 68, 220 (2001).

9. W. Reik, J. Walter, Nat. Rev. Genet. 2, 21 (2001).
10. A. Jost, S. Magre, R. Agelopoulou, Hum. Genet. 58, 59 (1981).

11. M. Buehr, S. Gu, A. McLaren, Development 117, 273 (1993).

12. G. Majdic, M. R. Millar, P. T. Saunders, J. Endocrinol. 147, 285 (1995).

13. H. O. Goyal et al., Anat. Rec. 249, 54 (1997).

14. W. R. Kelce, E. Monosson, M. P. Gamcsik, S. C. Laws, L. E. Gray Jr., Toxicol. Appl. Pharmacol. 126, 276 (1994).

15. A. M. Cummings, Crit. Rev. Toxicol. 27, 367 (1997). et al., Endocrinology 140, 5746 (1999).

17. W. R. Kelce, C. R. Lambright, L. E. Gray Jr., K. P. Roberts, Toxicol. Appl. Pharmacol. 142, 192 (1997).

18. J. S. Fisher, Reproduction 127, 305 (2004).

19. R. E. Chapin et al., Fundam. Appl. Toxicol. 40, 138 (1997).
20. A. S. Cupp et al., J. Androl. 24, 736 (2003).

21. M. Uzumcu, H. Suzuki, M. K. Skinner, Reprod. Toxicol. 18, 765 (2004).

22. B. S. Shi, Z. N. Cai, J. Yang, Y. N. Yu, Mutat. Res. 556, 1 (2004).

23. H. Dong et al., Biochemistry 43, 15922 (2004).

24. A. Tokumura, J. Cell. Biochem. 92, 869 (2004).

25. We acknowledge the technical contributions of I. Sadler-Riggleman, S. Rekow, and B. Johnston and the assistance of H. Suzuki with the methylation PCR procedure. This research was supported in part by a grant to M.K.S. from the U.S. Environmental Protection Agency's Science to Achieve Results (STAR) program involving endocrine disruptors. 


\title{
Science Supplemental Online Material
}

\section{Epigenetic Transgenerational Actions of Endocrine Disruptors and Male Fertility}

\author{
Matthew D. Anway, Andrea S. Cupp, Mehmet Uzumcu, Michael K. Skinner
}

doi: 10.1126/science. 1108190

\section{MATERIALS \& METHODS}

\section{A. In Vivo Procedures}

Gestating outbred Sprague-Dawley and inbred Fisher mother rats from timed pregnant colonies housed at the Washington State University Vivarium were given intraperitoneal injections of vinclozolin (100 $\mathrm{mg} / \mathrm{kg}$ per day) and methoxychlor $(200 \mathrm{mg} / \mathrm{kg} / \mathrm{day})$ from embryonic day 8-15 (E8-E15) of gestation ( $\mathrm{F}_{0}$ generation) as previously described $(\mathrm{S} 1)$. Sperm positive vaginal smear date being embryonic day 0 . Gestating control mothers received vehicle alone (i.e. sesame oil or DMSO). At least 6 lines (individual $\mathrm{F}_{0}$ injected females) were generated for controls and treated groups for these analyses. Male rats from control and treated groups were collected at P60-P180 for analyses. $\mathrm{F}_{1}$ treated males were bred to $F_{1}$ treated females to generate the $F_{2}$ treated generation and $F_{2}$ treated males were bred to $F_{2}$ treated females to generate the $F_{3}$ generation and the $F_{3}$ were bred in the same manner to generate the $\mathrm{F}_{4}$ generation. Rats for the control groups were bred in the same manner for all the generations. No inbreeding or sibling crosses were generated. The outcross group (VOC) was generated by breeding the $\mathrm{F}_{2}$ treated males with wild-type females (total of 6 litters) and reverse outcross group (RVOC) was generated by breeding $\mathrm{F}_{2}$ treated females with wild-type males (total of 3 litters). Control $(n=4)$ and treated ( $n=$ 4) male offspring from the $F_{1}$ generation from Fisher strain rats were collected at P6 for DNA methylation analysis, see below. All procedures have been approved by the Washington State University Animal Use and Care Committee. The numbers of animals used for replicates in the experiments (i.e., $n$ value) for vinclozolin treatment are as follows: $F_{1}$ (control 11, treated 12); $F_{2}$ (control 19, treated 30); $F_{3}$ (control 19, treated 26); $\mathrm{F}_{4}$ (control 15, treated 21); outcrosses (VOC 18, RVOC 6, wild-type controls 8). The methoxychlor treatment animal number was for $F_{1}$ (control 6, treated 9 ) and $F_{2}$ (control 16, treated 22). 


\section{B. Sperm Motility and Concentration Analysis}

Animals were sacrificed and cauda epididymal sperm motility was determined using cauda epididymal sperm. Briefly, the epididymis was dissected free of connective tissue and a small cut made to the cauda. The tissue was placed in $5 \mathrm{ml}$ of culture medium containing $0.1 \% \mathrm{BSA}$ for $10 \mathrm{~min}$ at $37^{\circ} \mathrm{C}$. An aliquot was placed on a pre-warmed slide and gently cover-slipped. The specimen was immediately examined using phase contrast microscopy with 100x magnification. All the motile sperm (i.e rapid progressive, slow progressive, and non-progressive) were counted according to WHO category (S2). Percent ratio of the motile sperm to the total number of sperm including immotile sperm was calculated. Epididymal sperm count was determined using the same epididymis sample according to a previously described method with some modifications $(S 3)$.

\section{Histology}

Tissues were fixed in Bouin's (Sigma, St. Louis, MO), embedded in paraffin, sectioned and then sections stained with hematoxylin and eosin according to standard procedures. The Center for Reproductive Biology, Histology Core Laboratory assisted with these procedures. The animal numbers are $n=50$ for vinclozolin treatment and $n=42$ for controls.

\section{Detection of Cell Apoptosis}

To detect apoptotic cells in testis sections, the Fluorescein In Situ Cell Death Detection Kit (Roche Applied Science, Indianapolis, IN) was utilized (S1). This system measures the fragmented DNA from apoptotic cells by enzymatically incorporating fluorecein-12-dUTP at the 3'-OH DNA ends using the enzyme terminal deoxynucleotidyl transferase which forms a polymeric tail using the principle of the TUNEL assay. Fluorescent apoptotic cells were imaged on a confocal microscope and number of apoptotic cells per testis cross section determined. A minimum of $n=8$ for vinclozolin and $n=6$ for controls for each generation was used. All cross sections used for TUNEL analysis had normal testis morphology.

\section{E. DNA Methylation Assays}

The methylation status of DNA isolated from control and endocrine disruptor treated P6 testis or epididymal sperm was determined using a combination of methylation sensitive restriction enzymes and a PCR approach previously described $(S 4, S 5)$. Briefly, isolated 
genomic DNA was incubated and digested with Rsa I with either methylation-sensitive (Hpa II) or insensitive (Msp I) restriction enzymes, followed by PCR with 10 primer sets designed to amplify methylation sites. PCR products were electrophoretically separated and visualized by SYBR green staining (Molecular Probes, Eugene, OR) and effects of endocrine disruptors determined by the presence or absence of specific bands. The PCR products of interest were isolated, cloned and sequenced to determine chromosomal location using BLAST Genbank analysis $(S 6, S 7)$. P6 testis analysis was repeated using 4 animals from different litters.

\section{F. Bisulfite Sequencing}

Genomic DNA was isolated from $\mathrm{F}_{1}-\mathrm{F}_{4}$ sperm samples using the DNeasy Tissue Kit (Qiagen, Valencia, CA) and digested with Rsa I. Genomic DNA was then treated with sodium bisulfite following methods previously established (S8). Briefly, $5 \mu \mathrm{g}$ of digested DNA was denatured in $\mathrm{NaOH}$ then treated with $4.0 \mathrm{M}$ sodium metabisulfite $\mathrm{pH} 5.0$ for 16 hours at $55^{\circ} \mathrm{C}$. Following desalting, DNA was desulfonated with $\mathrm{NaOH}$, neutralized and precipitated with ammonium acetate and ethanol. DNA samples were resuspended in 30 $\mu \mathrm{L}$ of $1 \mathrm{mM}$ Tris buffer, $\mathrm{pH}$ 8.0. Sequence specific primers were generated to amplify the region of interest. PCR products were cloned into pGEMT Easy vector (Promega Corporation) and sequenced. Approximately 35 clones from each PCR product were sequenced to determine the methylation state of the $\mathrm{CpG}$ sites. Confirming altered methylation states involved comparisons of the sequences following the bisulfite treatment. The primers for the lysophospholipase PCR bisulfite analysis were 5'GGT ATA TAT AGA GGA AGG TAG GTA GG3' and 5'TAA AAA CCT CCA AAA AAC

\section{AAA CAC T3'.}

\section{G. Statistical Analysis}

The data from apoptotic cell numbers, sperm motility and sperm count were analyzed using a SAS program. The values were expressed as the mean \pm SEM. Statistical analysis was performed and the difference between the means of treatments and respective controls was determined using two-way Analysis of Variance (ANOVA). Analysis performed with JMP v3.1.6, SAS Institute Inc, Cary, NC. In vivo experiments were repeated with 6-30 individuals for each data point. A statistically significant difference was confirmed at $P<$ 0.05 . 


\section{References and Notes}

S1. A. S. Cupp et al., J Androl 24, 736 (2003).

S2. U. Kvist, L. Bjorndah., Manual on Basic Semen Analysis, ESHRE monographs (ESHRE and Oxford Univ., Germany, Rev. ed., 2002), pp. 14-17.

S3. $\quad$ G. T. Taylor, J. Weiss, T. Frechmann, J. Haller, J Reprod Fertil 73, 323 (1985).

S4. C. X. Zhong, M. J. Mass, Toxicol Lett 122, 223 (2001).

S5. G. Liang et al., Genomics 53, 260 (1998).

S6. A. K. Hotchkiss, J. S. Ostby, J. G. Vandenburgh, L. E. Gray, Jr., Environ Health Perspect 110 (suppl. 3), 435 (2002).

S7. I. P. Kapoor, R. L. Metcalf, R. F. Nystrom, G. K. Sangha, J Agric Food Chem 18, 1145 (1970).

S8. M. Frommer et al., Proc Natl Acad Sci U S A 89, 1827 (1992). 
A.

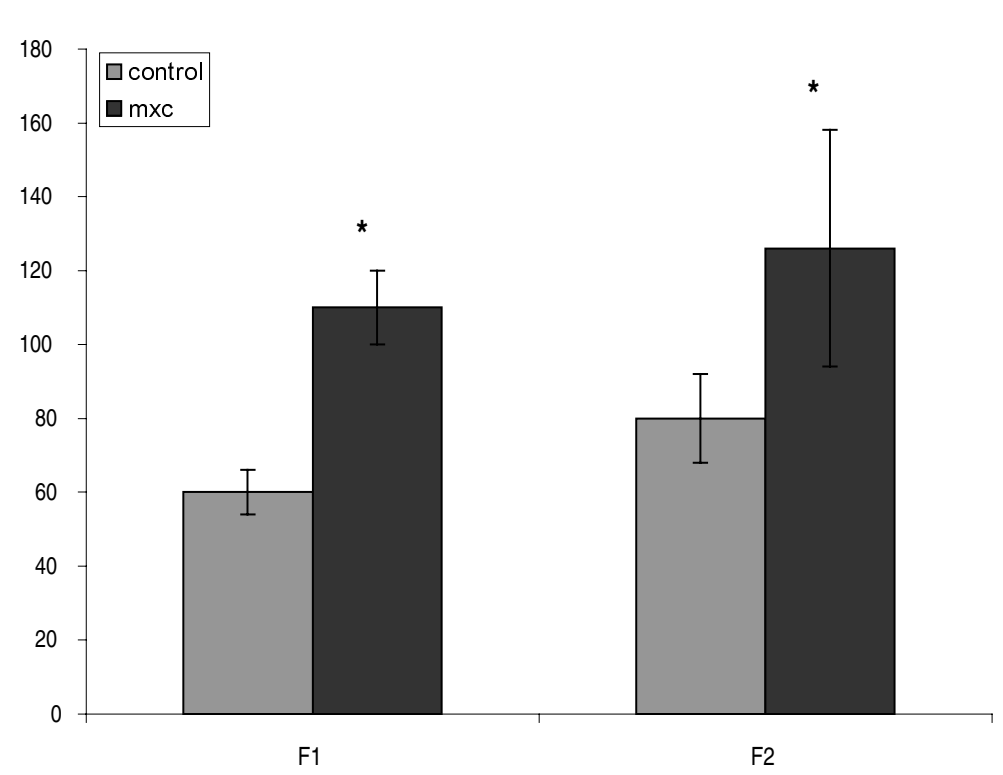

e Material

et al., p. 5

B.

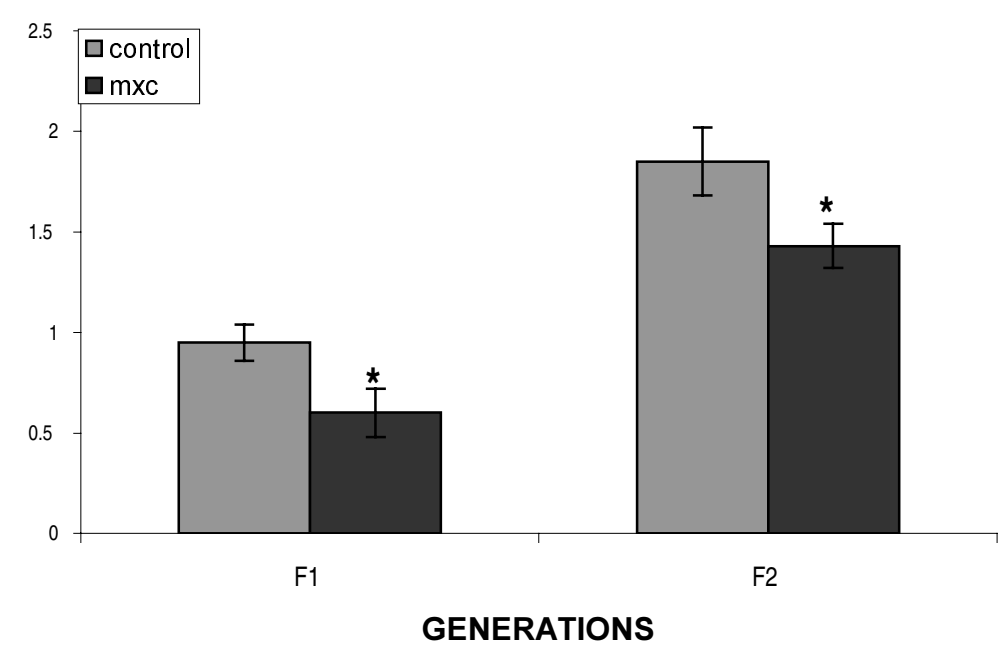

C.

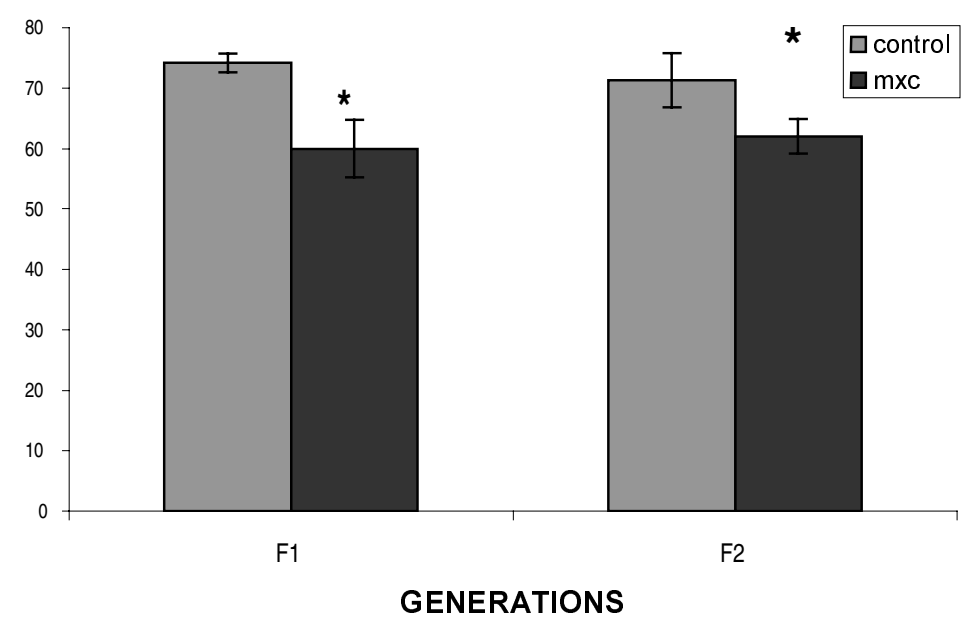

Fig. S1. Transgenerational phenotype after methoxychlor treatment. (A) Spermatogenic cell apoptosis. (B) epididymal sperm counts. (C) Epididymal sperm motility in P60-P120 control and treated Fisher rats in $\mathrm{F}_{1}$ and $\mathrm{F}_{2}$ generations. Statistically significantly differences between control and treated animals within a generation are indicated by $(*)$ for $P<0.05$. The number of animals for each bar ranged between 6 and 12 . 
Science Supporting Online Material Anway et al., p. 6

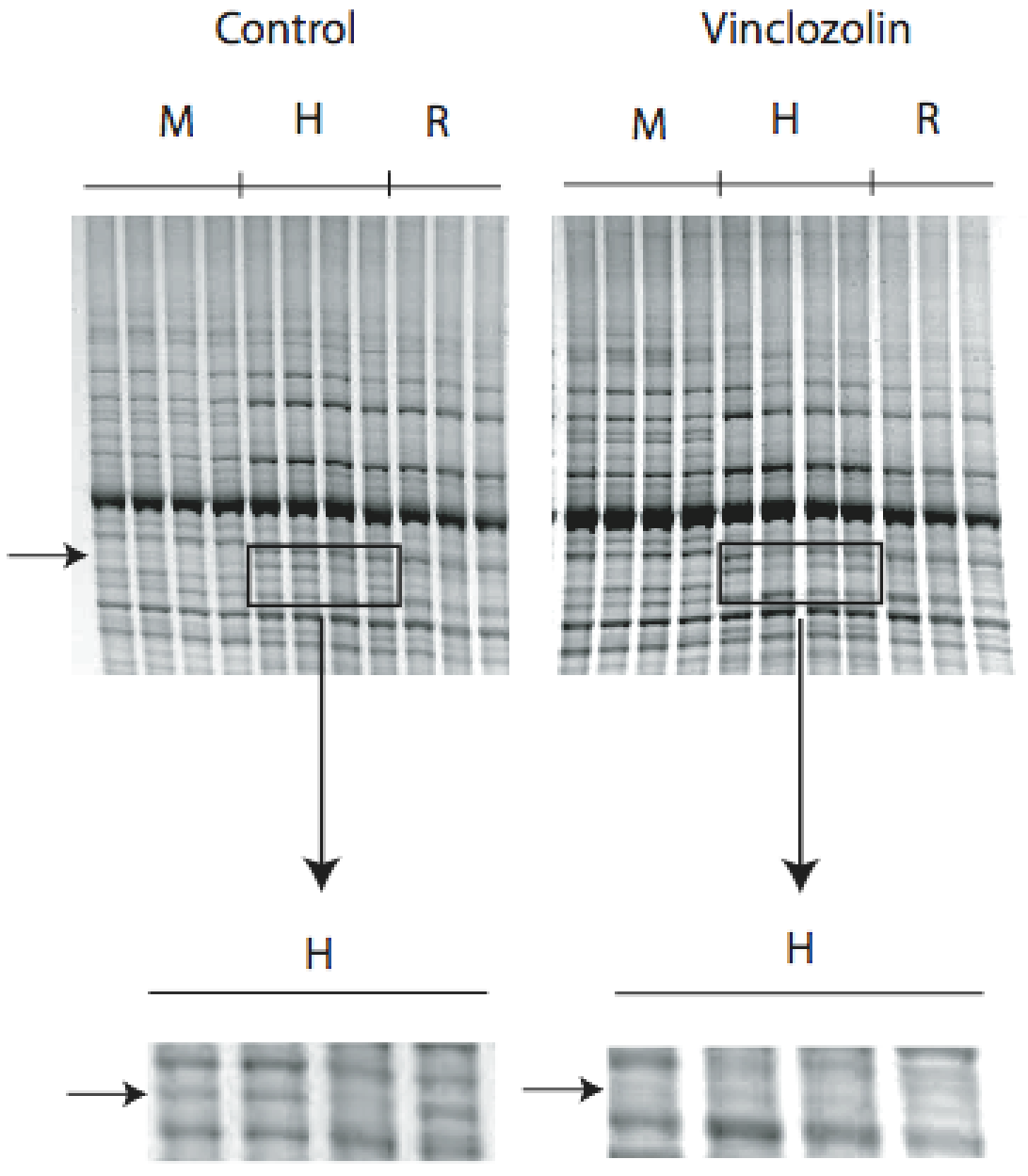

Supplemental Figure S2. DNA methylation analysis from control and treated P6 testis. Representative gel image of the PCR based methylation sensitive H-Hpall+Rsal $(H)$ and insensitive Mspl + Rsal (M), and Rsal (R) as control. restriction enzyme digest analysis with representative band (arrow) (200bp) affected by in vivo exposure to vinclozolin shown. Postnatal day 6 (P6) testis from control (control) and vinclozolin (treated) treatment presented. Each lane represents the analysis of a different animal P6 testis $(n=4)$. 
Science Supporting Online Material

Anway et al., p. 7

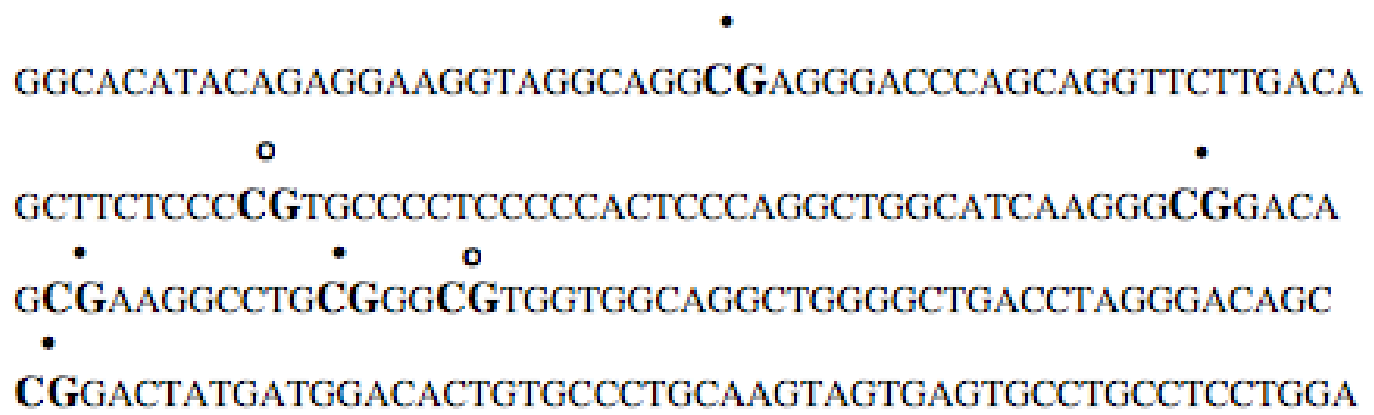

GGTCCTCA

Supplemental Figure S3. DNA sequence from the lysophospholipase (LPL) gene with altered methylation pattern. The LPL genomic sequence (accession \#NW_047762.1) from base pairs 32270685 to 32270889 . Methylated cytosine residues (Bold CG) present in both control and vinclozolin generation animal sperm are marked with a closed circle $(\cdot)$ and those altered in vinclozolin $\mathrm{F} 1-\mathrm{F} 4$ generation sperm samples are marked with an open circle $\left({ }^{\circ}\right)$. 